\title{
CHARGE CONJUGATION AND MAIORANA SPINORS IN DIMENSION 8
}

\author{
PERTTI LOUNESTO \\ Institute of Mathematics \\ Helsinki University of Technology \\ SF-02150 Espoo, Finland
}

\begin{abstract}
It is a common belief among theoretical physicists that the charge conjugation of the Dirac equation has an analogy in higher dimensional space-times so that in an 8-dimensional space-time there would also be Maiorana spinors as eigenspinors of a charge conjugation, which would swap the sign of the electric charge of the Dirac equation. This article shows that this mistaken belief is based on inadequate distinction between two kinds of charge conjugation: the electric conjugation swapping the sign of the Dirac equation and the Maiorana conjugation fixing the real subalgebra (of the complexification of the Clifford algebra of the space-time). Only the latter conjugation has Maiorana spinors as its eigenspinors.
\end{abstract}

1. Introduction. All books and articles on Maiorana spinors in higher dimensions tacitly assume that Maiorana spinors are eigenspinors of a charge conjugation which swaps the sign of a Dirac equation in that higher dimension. This article shows that it is necessary to make a distinction between two kinds of charge conjugation: the electric conjugation swapping the sign of the Dirac equation and the Maiorana conjugation fixing the real subalgebra (of the complexification of the Clifford algebra of the space-time). Only the latter conjugation has Maiorana spinors as its eigenspinors.

This misconception is sharpest in Budinich \& Trautman 1988, and we shall relate our discussion to their book. However, the misconception is tacit in many other books dealing with the Dirac equation, charge conjugation and Maiorana spinors. Benn \& Tucker 1987 do not actually make the error, although they do not make a proper distinction either (between the electric conjugation and the Maiorana conjugation).

There are also books which elude this error by dealing only with some parts of the topic. Delanghe \& Sommen \& Souček 1992 deal with the Dirac equation and Maiorana spinors, but do not discuss on the charge conjugation. Harvey 1990 deals with the charge conjugation and Maiorana spinors (observe differences in terminology), but does not

1991 Mathematics Subject Classification: Primary 81R25; Secondary 53A50, 32L25.

The paper is in final form and no version of it will be published elsewhere. 
discuss on the Dirac equation.

2. Minkowski space $\mathbb{R}^{1,3}$ with $C l_{1,3} \simeq \operatorname{Mat}(2, \mathbb{H})$. In the signature $\mathbb{R}^{1,3}$ we have the Dirac equation $i \partial \psi-e \mathbf{A} \psi=m \psi$ or $\gamma^{\mu}\left(i \partial_{\mu} \psi-e \mathbf{A}_{\mu}\right) \psi=m \psi$ where

$$
\gamma_{0}=\gamma^{0}=\left(\begin{array}{cc}
1 & 0 \\
0 & -1
\end{array}\right) \quad \text { and } \quad \gamma_{k}=-\gamma^{k}=\left(\begin{array}{cc}
0 & -\sigma_{k} \\
\sigma_{k} & 0
\end{array}\right) \quad \text { for } \quad k=1,2,3
$$

and where $\sigma_{1}, \sigma_{2}, \sigma_{3}$ are the Pauli spin matrices (Budinich \& Trautman 1988 page 3 line 4 formula 1.5 ). Usually $\psi \in \mathbb{C}^{4}$ is a column spinor but I shall regard the spinor $\psi$ as a $4 \times 4$-matrix with only the first column being nonzero, that is, $\psi \in \operatorname{Mat}(4, \mathbb{C}) f$, where $f=\frac{1}{2}\left(1+\gamma_{0}\right) \frac{1}{2}\left(1+i \gamma_{1} \gamma_{2}\right)$. Maiorana spinors $\psi$ are eigenspinors $\mathcal{C}(\psi)= \pm \psi$ of the charge conjugation operator $\mathcal{C}$ which is defined by

$$
\mathcal{C}(\psi)=-i \gamma_{2} \psi^{*} \quad \text { complex conjugate } \psi^{*} \text { taken in } \operatorname{Mat}(4, \mathbb{C})
$$

or

$$
\mathcal{C}(\psi)=\hat{\psi}^{*} \gamma_{1} \quad \text { complex conjugate } \psi^{*} \text { taken in } \mathbb{C} \otimes C l_{1,3}
$$

( $u \rightarrow \hat{u}$ means the grade involution). The charge conjugated spinor $\psi_{C}=\mathcal{C}(\psi)$ satisfies the Dirac equation $i \partial \psi_{C}+e \mathbf{A} \psi_{C}=m \psi_{C}$. The charge conjugation is anti-linear $\mathcal{C} i=-i \mathcal{C}$, involutory $\mathcal{C}^{2}=I$ and satisfies $\mathcal{C}(\mathbf{A} \psi)=-\mathbf{A} \mathcal{C}(\psi)$. In the sequel I will abbreviate the last equation to $\mathcal{C} \mathbf{A}=-\mathbf{A} \mathcal{C}$.

The normalized bilinear covariants of $\psi$ result in the unit velocity $u=u_{0} \gamma^{0}+u_{1} \gamma^{1}+$ $u_{2} \gamma^{2}+u_{3} \gamma^{3}$, which is time-like $u^{2}=1>0\left(u^{2}=u_{0}^{2}-u_{1}^{2}-u_{2}^{2}-u_{3}^{3}\right)$ and is related to the velocity $v<c$ of a real particle by

$$
u_{0}=\frac{1}{\sqrt{1-\frac{v^{2}}{c^{2}}}} .
$$

For an imaginary particle, or superluminary tachyon, $v>c$ and $u$ is purely imaginary so that $i u$ is space-like $(i u)^{2}=-1<0$.

3. Opposite metric $\mathbb{R}^{3,1}$ with $C l_{3,1} \simeq \operatorname{Mat}(4, \mathbb{R})$. In the opposite metric $\mathbb{R}^{3,1}$ the Dirac equation is $\partial \psi+i e \mathbf{A} \psi=m \psi$. This form of the Dirac equation guarantees that the velocity $u=u_{0} \mathbf{e}^{0}+u_{1} \mathbf{e}^{1}+u_{2} \mathbf{e}^{2}+u_{3} \mathbf{e}^{3}$ is real and time-like $u^{2}=-1<0$ $\left(u^{2}=-u_{0}^{2}+u_{1}^{2}+u_{2}^{2}+u_{3}^{3}\right)$ for a real particle. The matrix representation

$$
\mathbf{e}_{0}=-\mathbf{e}^{0}=\left(\begin{array}{cc}
i & 0 \\
0 & -i
\end{array}\right) \quad \text { and } \quad \mathbf{e}_{k}=\mathbf{e}^{k}=\left(\begin{array}{cc}
0 & \sigma_{k} \\
\sigma_{k} & 0
\end{array}\right) \quad \text { for } \quad k=1,2,3
$$

is obtained by the choice $f=\frac{1}{2}\left(1-i \mathbf{e}_{0}\right) \frac{1}{2}\left(1-i \mathbf{e}_{1} \mathbf{e}_{2}\right)$ and results in the charge conjugation

$$
\mathcal{C}(\psi)=-i \mathbf{e}_{0} \mathbf{e}_{2} \psi^{*} \quad \text { complex conjugate } \psi^{*} \text { taken in } \operatorname{Mat}(4, \mathbb{C})
$$

or

$$
\mathcal{C}(\psi)=\psi^{*} \mathbf{e}_{0} \mathbf{e}_{1} \quad \text { complex conjugate } \psi^{*} \text { taken in } \mathbb{C} \otimes C l_{3,1} .
$$

It is possible to represent $C l_{3,1}$ by real matrices as follows

$$
\epsilon_{1}=\left(\begin{array}{cc}
\sigma_{3} & 0 \\
0 & -\sigma_{3}
\end{array}\right), \quad \epsilon_{2}=\left(\begin{array}{cc}
\sigma_{1} & 0 \\
0 & \sigma_{1}
\end{array}\right), \quad \epsilon_{3}=\left(\begin{array}{cc}
0 & \sigma_{3} \\
\sigma_{3} & 0
\end{array}\right), \quad \epsilon_{4}=\left(\begin{array}{cc}
-i \sigma_{2} & 0 \\
0 & -i \sigma_{2}
\end{array}\right)
$$


obtained by the choice $f=\frac{1}{2}\left(1+\mathbf{e}_{1}\right) \frac{1}{2}\left(1+\mathbf{e}_{2} \mathbf{e}_{4}\right)$. This results in $\mathcal{C}(\psi)=\psi^{*}$ for both $\mathbb{C} \otimes \operatorname{Mat}(4, \mathbb{R}) \simeq \operatorname{Mat}(4, \mathbb{C})$ and $\mathbb{C} \otimes C l_{3,1}$.

For either matrix representation the charge conjugation is anti-linear $\mathcal{C} i=-i \mathcal{C}$, involutory $\mathcal{C}^{2}=I$ and satisfies $\mathcal{C} \mathbf{A}=\mathbf{A C}$.

4. Conjugations in general. I regard the spinor space $S$ as a minimal left ideal $S=\left(\mathbb{C} \otimes C l_{3,1}\right) f$, where the idempotent $f$ is primitive in the complexified Clifford algebra $\mathbb{C} \otimes C l_{p, q}$. A real linear transformation $\mathcal{C}: S \rightarrow S$ is called a conjugation if it is antilinear $\mathcal{C} i=-i \mathcal{C}$ and satisfies $\mathcal{C}^{2}= \pm I$. In principle, a charge conjugation could be either involutory $\mathcal{C}^{2}=I$ or satisfy $\mathcal{C}^{2}=-I$. However, in the latter case anti-linearity implies $\frac{1}{2}(1 \pm i \mathcal{C})^{2}= \pm i \mathcal{C}$, and so the operations $\frac{1}{2}(1 \pm i \mathcal{C})$ do not project complex subspaces of the spinor space $S$.

For this reason it is appropriate to omit the 'quaternionic' charge conjugations $\mathcal{C}^{2}=$ $-I$, and I will exclude them, because a quaternionic conjugation $\mathcal{C}$, such that $\mathcal{C}^{2}=-I$, does not have real eigenvalues and because Budinnich \& Trautman 1988 attach Maiorana spinors, with eigenvalues \pm 1 , only to the involutory charge conjugations $\mathcal{C}^{2}=I$ (this is implied by page 95 , lines 5,8 and 22 ).

On pages 90-97 Budinich \& Trautman 1988 adopt, unintentionally, two different approaches to charge conjugation, which are not compatible with each other. In order to make a distinction between the two concepts of charge conjugation I will, from now on, call one of them the Electric conjugation $\mathcal{E}$ of the Dirac equation and the other the Maiorana conjugation $\mathcal{M}$ with Maiorana spinors as eigenspinors.

5. Electric conjugation in higher dimensions. On page 94 Budinich \& Trautman 1988 consider the Dirac equation not only in the Minkowski space $\mathbb{R}^{3,1}$ or $\mathbb{R}^{1,3}$ but also in higher dimensions. Budinich \& Trautman 1988 refer to 'real particles of momentum $p$ ' whereby they focus on the Lorentz signatures $\mathbb{R}^{n-1,1}$ or $\mathbb{R}^{1, n-1}$. [The momentum $p$ of a particle is apparently a time-like vector.]

The electric conjugation $\mathcal{E}$ should transform the Dirac equation (page 94 formulas $7.25 \mathrm{i}$ and 7.25$)$

to the following form ${ }^{1}$

$$
\mathbb{R}^{1, n-1} \quad i \partial \psi-e \mathbf{A} \psi=m \psi \quad \partial \psi+i e \mathbf{A} \psi=m \psi \quad \mathbb{R}^{n-1,1}
$$

$$
i \partial \psi_{C}+e \mathbf{A} \psi_{C}=m \psi_{C} \quad \partial \psi_{C}-i e \mathbf{A} \psi_{C}=m \psi_{C}
$$

(page 95 lines 1-3). This change of sign is assumed to be accomplished by a real linear transformation of spinors $\mathcal{E}: S \rightarrow S, \psi \rightarrow \psi_{C}$ which is anti-linear $\mathcal{E}(i \psi)=-i \mathcal{E}(\psi)$ and involutory $\mathcal{E}(\mathcal{E}(\psi))=\psi$. An anti-linear transformation swaps the signs as designated above if and only if the following is satisfied (consequences of Budinich \& Trautman 1988 page 95 lines 1-3)

$$
\mathbb{R}^{1, n-1} \quad \mathcal{E}(\mathbf{A} \psi)=-\mathbf{A} \mathcal{E}(\psi) \quad \mathcal{E}(\mathbf{A} \psi)=\mathbf{A} \mathcal{E}(\psi) \quad \mathbb{R}^{n-1,1} .
$$

\footnotetext{
${ }^{1}$ Note that the electric conjugation has no effect on the scalar $e$, that is, $\mathcal{E}(e \psi)=e \mathcal{E}(\psi)$, but rather affects the spinor $\psi$. Also note that I use the opposite sign of $e$ in comparison with Budinich \& Trautman, and that I also present 7.25i before 7.25.
} 
To summarize, Budinich \& Trautman 1988 look for higher-dimensional analogies of the Dirac particle - by implication in the Lorentz signatures with a time-like momentum $p=i \partial$ - and refer to an electric conjugation which is anti-linear $\mathcal{E} i=-i \mathcal{E}$, involutory $\mathcal{E}^{2}=I$ and such that (gauge $\partial \rightarrow \partial+i e \mathbf{A}$ )

$$
\begin{array}{rl}
C l_{1, n-1} & C l_{n-1,1} \\
p^{2}-m^{2}=0 & p^{2}+m^{2}=0 \\
\left(\partial^{2}+m^{2}\right) \psi=0 & \left(\partial^{2}-m^{2}\right) \psi=0 \\
i \partial \psi-e \mathbf{A} \psi=m \psi & \partial \psi+i e \mathbf{A} \psi=m \psi \\
\underline{\mathcal{E} \mathbf{A}=-\mathbf{A} \mathcal{E}} & \underline{\mathcal{E} \mathbf{A}=\mathbf{A} \mathcal{E}}
\end{array}
$$

The Dirac equation could be generalized from the Lorentz spaces to arbitrary signatures.

6. Maiorana conjugation has Maiorana spinors as eigenspinors. Budinich \& Trautman 1988 define the Maiorana conjugation on page 91 line 1 formula 7.19 , page 91 lines 6-7, page 95 lines 5/8 formulas 7.26i/7.26 and page 95 line -5 . Their definition results in a real linear transformation $\mathcal{M}$ of spinors which is anti-linear $\mathcal{M} i=-i \mathcal{M}$, involutory $\mathcal{M}^{2}=I$ and such that $[$ here $\mathcal{A}(d)=\operatorname{Mat}(d, \mathcal{A})]$

$\begin{array}{clclcl} & & p-q & C l_{1, n-1} & n \\ \mathcal{M A}= \pm \mathbf{A} \mathcal{M} & \mathcal{M} \simeq C, C \Gamma & 0 & \mathbb{R}(2) & 2 & \\ \mathcal{M A}=-\mathbf{A} \mathcal{M} & \mathcal{M} \simeq C \Gamma & 6 & \mathbb{H}(2) & 4 & \\ & & 4 & \mathrm{H}(4) & 6 & \\ \mathcal{M A}=\mathbf{A} \mathcal{M} & \mathcal{M} \simeq C & 2 & \mathrm{R}(16) & 8 & \\ n & C l_{n-1,1} & p-q & & & \\ 2 & \mathbb{R}(2) & 0 & \mathcal{M} \simeq C C \Gamma & \mathcal{M A}= \pm \mathbf{A} \mathcal{M} \\ 4 & \mathrm{R}(4) & 2 & & \mathcal{M} \simeq C & \mathcal{M A}=\mathbf{A} \mathcal{M} \\ 6 & \mathrm{H}(4) & 4 & & & \\ 8 & \mathrm{H}(8) & 6 & & \mathcal{M} \simeq C \Gamma & \mathcal{M A}=-\mathbf{A} \mathcal{M}\end{array}$

In the signatures $p-q=4 \bmod 8$ there are no Maiorana spinors (page 97 line 12), that is, there is no involutive Maiorana conjugation (so exclude the case $p-q=4 \bmod 8$ ). The notions $\mathcal{M} \simeq C$ and $\mathcal{M} \simeq C \Gamma$ above mean $\mathcal{M}(\psi)=[C(\psi)]^{*}$ (page 95 line 5 formula 7.26) and $\mathcal{M}(\psi)=[C \Gamma(\psi)]^{*}$ (page 95 line 8 formula 7.26i). The communication relations on page 91 line 1 formula 7.19 together with page 95 line 5 (and 8) mean that

$$
[C(\mathbf{A} \psi)]^{*}=C^{*} \mathbf{A}^{*} \psi^{*}=\mathbf{A} C^{*} \psi^{*}=\mathbf{A}[C(\psi)]^{*} \quad \text { in } \operatorname{Mat}(d, \mathbb{C})
$$

which together with $\mathbf{A} \Gamma=-\Gamma \mathbf{A}$ implies (the case $p-q=0$ is irrelevant)

$$
\begin{array}{cccc}
\mathcal{M A}=\mathbf{A} \mathcal{M} & \text { for } & \mathbf{A} \in \mathbb{R}^{p, q}, & p-q=0,2 \bmod 8 \\
\mathcal{M A}=-\mathbf{A} \mathcal{M} & \text { for } & \mathbf{A} \in \mathbb{R}^{p, q}, & p-q=0,6 \bmod 8
\end{array}
$$

after recalling that Budinich \& Trautman 1988 define on page 95 lines 8,-5,-1 the Maiorana conjugation to be $\mathcal{M} \simeq C \Gamma$ in the signature $p-q=6 \bmod 8$. 
7. Electric conjugation $\neq$ Maiorana conjugation. The formulas underlined ( $=$ consequences of page 91 line 1 formula 7.19 and page 95 line -5 ) show that the Maiorana conjugation $\mathcal{M}$ (of Budinich \& Trautman) is not in accordance with their electric conjugation $\mathcal{E}$ (of Budinich \& Trautman) in dimension 8. The Maiorana conjugation transforms the Dirac equation ( $\partial$ behaves like a vector $\mathbf{A}$ with respect to commutations with $\mathcal{M}$ ):

$$
\begin{aligned}
& i \partial \psi-e \mathbf{A} \psi=m \psi \quad \text { in } \quad \mathbb{R}^{1,7} \quad \text { to } \quad-i \partial \mathcal{M}(\psi)-e \mathbf{A} \mathcal{M}(\psi)=m \mathcal{M}(\psi), \\
& \partial \psi+i e \mathbf{A} \psi=m \psi \quad \text { in } \quad \mathbb{R}^{7,1} \quad \text { to } \quad-\partial \mathcal{M}(\psi)+i e \mathbf{A} \mathcal{M}(\psi)=m \mathcal{M}(\psi) .
\end{aligned}
$$

The electric charge is not conjugated according to the characterizations on page 94 and top three lines on page 95, which contradicts the theory of Budinich \& Trautman 1988 on the (electric) charge conjugation and Maiorana spinors. In fact, there is no electric (charge) conjugation in dimension 8.

\section{References}

[1] M. Benn and R. W. Tucker, An Introduction to Spinors and Geometry with Applications in Physics, Adam Hilger, Bristol 1987.

[2] P. Budinich and A. Trautman, The Spinorial Chessboard, Berlin 1988.

[3] A. Crumeyrolle, Orthogonal and Symplectic Clifford Algebras, Spinor Structures, Kluwer Academic, Dordrecht 1990.

[4] R. Delanghe, F. Sommen, and V. Souček, Clifford Algebra and Spinor Valued Functions: A function theory for the Dirac operator, Kluwer Academic, Dordrecht 1992.

[5] F. R. Harvey, Spinors and Calibrations, Academic Press, San Diego 1990. 